\title{
Elektrische Doppelbrechung von Tabakmosaikvirus-Lösungen in Abhängigkeit von der Erwärmung*
}

\author{
Von W. PawlitscheK ** \\ Aus der Biologischen Zentralanstalt der Deutschen Akademie der Landwirtschaftswissenschaften \\ zu Berlin, Institut für Phytopathologie Aschersleben (Direktor : Prof. Dr. M. KLInkowskr) \\ und dem Institut für experimentelle Physik der Martin-Luther-Universität Halle-Wittenberg \\ (Direktor: Prof. Dr. W. Messerschmidt) \\ (Z. Naturforschg. 15 b, 153-163 [1960] ; eingegangen am 14. September 1959)
}

\begin{abstract}
Es werden zunächst die theoretischen Grundlagen über die optische Anisotropie von Lösungen stäbchenförmiger Teilchen sowie die spezielle Theorie zur Orientierungs-Doppelbrechung und zur Versuchsdurchführung dargestellt. Die Anwendung beschränkt sich auf die Relaxations-Erscheinungen der Doppelbrechung einer TMV-Lösung in bezug auf ein elektrisches Wechselfeld in Abhängigkeit von der Erwärmung. Bei einer zeitlich fast linear zunehmenden Erwärmung $\Delta T / \Delta t=1,5 \mathrm{grad} / \mathrm{min}$ konnten für die Phasenverschiebungen der Doppelbrechung charakteristische und reproduzierbare Kurvenverläufe ermittelt werden. Diese Kurven legten eine Einteilung in 2 Zonen nahe, deren Grenze bei 50,5-52,5 ${ }^{\circ} \mathrm{C}$ lag, die gleichzeitig den Beginn der Protein-Denaturierung darstellt. Weitere Ergebnisse, die teilweise neue Fragen aufgeworfen haben, werden diskutiert. Ferner wird mit Hilfe der elektronenmikroskopischen Teilchenvermessung die Rotationsdiffusions-Konstante berechnet und mit dem Wert, der sich aus der Phasenverschiebung des K e r r - Effektes ergibt, verglichen. Es findet sich eine Übereinstimmung innerhalb der Fehlergrenzen. Das elektronenoptische Bild des TMV nach der Denaturierung läßt kugelförmige Teilchen erkennen, zu denen das Virusprotein koaguliert ist.
\end{abstract}

In der vorliegenden Arbeit sollte untersucht werden, inwieweit sich der Prozeß der Hitzedenaturierung des Tabakmosaikvirus-Proteins * auf Relaxations-Erscheinungen beim Kerr-Effekt von TMV-Lösungen auswirkt.

Arbeiten über den elektrooptischen Effekt von TMVLösungen liegen bereits von einigen Autoren vor. Als erste berichteten zunächst TAKAHASH und Rawlins (1932, 1933) $)^{1,2}$ über Strömungsdoppelbrechung von Preßsaft aus TMV-kranken Pflanzen. Durch Beseitigung der Verunreinigungen konnte dieser Effekt noch verstärkt werden. Ebenfalls vor mehr als zwanzig Jahren berichteten Bawnen, Pirie, Bernal und Fankuchen $(1936)^{3}$ über den optisch anisotropen Charakter von nach Angaben von Stanley $(1936)^{4}$ gereinigten TMVLösungen, wenn diese durch eine Kapillare strömten oder sich in einem elektrischen Feld befanden. Diese Autoren haben somit den Kerr-Effekt bei TMVLösungen als erste qualitativ erfaßt.

KaUSCHE und Lwowski (1951) ${ }^{5}$ haben diesen Effekt zur Bestimmung des Aggregationszustandes einiger Stämme des TMV herangezogen. LAUfFer (1939) ${ }^{6}$ stellte bei ähnlichen Untersuchungen fest, daß die elektrische Doppelbrechung nicht dem üblichen Ke r r Gesetz folgt; allerdings stehen hierzu die Arbeiten von

* Auszug aus Diss., Halle/S. 1959.

** Anschrift des Verf.: Wernigerode/Harz, Büchtingenstr. 36, Deutschland.

* Im weiteren Verlauf der Arbeit wird für Tabakmosaikvirus die heute gebräuchliche Abkürzung "TMV“ benutzt.

1 W. N. Takahashi u. T. E. Rawlins, Proc. Soc. exp. Biol. Med. 30, 155 [1932], zitiert nach Lauffer und Stanley, 1939.

2 W. N. Takahashi u. T. E. Rawlins, Science [New York] 77, 26 [1933], zitiert nach Lauffer und Stanlex, 1939.

3 F. C. Bawden, N. W. Pirie, J. D. Bernal u. I. Fankuchen, Nature [London] 138, 1051 [1936].
O'Konski und Haltner (1956, 1957) 7,8 im Widerspruch. Diese Autoren fanden bei Anwendung eines Wechselfeldes eine lineare Zunahme der Doppelbrechung mit dem Quadrat der Feldstärke, wie es das K e r r-Gesetz fordert. Norman und Field (1957 a und b) ${ }^{9,10}$ konnten bei ähnlichen Versuchen diesen Befund für kleine Feldstärken bestätigen. Bei höheren Feldern dagegen, etwa $600 \mathrm{~V} / \mathrm{cm}$, wenn die Orientierungsenergie der Teilchen so groß war wie ihre thermische Energie, strebte die Doppelbrechung einem Sättigungswert zu.

Allgemein herrscht heute die Ansicht vor, daß das TMV keinen meßbaren permanenten Dipol aufweist, sondern daß im elektrischen Feld durch Ionenverschiebung lediglich ein elektrischer Dipol influenziert wird. Eine Reihe von Problemen ist jedoch noch ungelöst, und die Diskussion hierüber befindet sich im Fluß.

\section{Theoretischer Teil}

\section{Allgemeines zur Anisotropie}

Die Ladungsträger, aus denen Atome und Moleküle aufgebaut sind, sind nicht miteinander starr

4 W. M. Stanley, Phytopathology 26, 305 [1936].

5 G. A. Kausche u. W. Lwowski, Z. Naturforschg. 6 b, 60 [1951].

6 M. A. Lauffer, J. Amer. chem. Soc. 61, 2412 [1939].

7 C. T. O'Konsir u. A. J. Haltner, J. Amer. chem. Soc. 78, 3604 [1956].

8 C. T. O'Konski u. A. J. Haltner, J. Amer. chem. Soc. 79, 5634 [1957].

9 A. Normann u. A. J. Field, jr., Arch. Biochem. Biophysics 70, 257 [1957 a].

10 A. Normann u. J. A. Field, jr., Arch. Biochem. Biophysics 71, 170 [1957]. 
verbunden. Unter dem Einfluß eines elektrischen Feldes $₹$ können daher die Ladungsträger (Elektronen- bzw. Ionenwolke) gegeneinander verschoben werden, so daß die Schwerpunkte der positiven und negativen Ladungen nicht mehr zusammenfallen. Es bildet sich ein Dipol mit einem für das betreffende Molekül charakteristischen Moment $\mathfrak{p}$. Die Summe aller dieser elementaren Dipole ergibt das Moment $\mathfrak{S}$ des Einheitsvolumens des betrachteten Mediums. Man nennt $\mathfrak{P}$ auch „Verschiebungs-Polarisation“, und für sie gilt demnach die Beziehung:

$$
\mathfrak{P}=\sum_{1}^{N} \mathfrak{p}_{\mathrm{i}}=\sum_{1}^{N}\left(p_{x} \mathfrak{i}+p_{y} \dot{\mathfrak{i}}+p_{z} \mathfrak{k}\right)_{\mathrm{i}}
$$

( $\mathfrak{i}, \mathfrak{j}, \mathfrak{f}=$ Einheitsvektoren, $N=$ Anzahl der elementaren Dipole im betrachteten Volumenelement). Da die Polarisation in Richtung des Feldes erfolgt, ist die Isotropiebedingung $\bar{p}_{x}=\bar{p}_{y}=\bar{p}_{z}=0$ im allgemeinen nicht erfüllt; bei optischen Frequenzen tritt dann Doppelbrechung auf.

Ferner gilt nach der Maxwellschen Theorie für die Polarisation die Gleichung

$$
\mathfrak{P}=\vartheta-\vartheta_{0}=\left(\varepsilon-\varepsilon_{0}\right) \mathfrak{E}=\chi \mathfrak{E},
$$

in der $\vartheta$ die „dielektrische Verschiebung“ des Mediums unter dem Einfluß des Feldes $₹$ darstellt und $\vartheta_{0}$ der entsprechende Vakuumanteil ist; $\varepsilon-\varepsilon_{0}=\chi$ ist die "dielektrische Suszeptibilität" und $\varepsilon$ und $\varepsilon_{0}$ sind die Dielektrizitäts-Konstanten des Mediums bzw. Vakuums. Im Falle der Isotropie ist der Faktor $\chi$ ein Skalar, liegt dagegen Anisotropie vor, so wird er zu einem Tensor, der bei geeigneter Drehung des Koordinatensystems durch eine Diagonalmatrix dargestellt werden kann. Obige Gleichung lautet dann:

$$
\mathfrak{P}_{\mathrm{i}}=\sum_{1}^{3} \chi_{\mathrm{i}} \mathfrak{E}_{\mathrm{i}}
$$

Wegen der M a x w ell schen Beziehung

$$
n^{2}=\frac{\varepsilon}{\varepsilon_{0}} \cdot \frac{\mu}{\mu_{0}}
$$

( $n=$ Brechzahl, $\mu \approx \mu_{0}=$ magnetische Permeabilität)

11 H. Benort, Ann. Physique, 12e Série, 6, 561 [1951].

12 H. Benort, J. Chim. physique 49, 518 [1952].

13 H. A. Stuart, Elektrischer K e r r - Effekt (Elektr. Doppelbrechung), in: Handb. chem. Physik, Bd. 10/III, S. 27 bis $104,1939$.

14 H. A. Stuart, Elektrische Doppelbrechung, optische Anisotropie und Molekülstruktur, in: Die Phys. d. Hochpolymeren, Bd. I, S. 416-458, 1953. kann man für alle $\varepsilon$-Werte auch die entsprechenden $n^{2}$-Werte einsetzen.

Bezeichnet man mit $n_{\mathrm{p}}=n_{3}$ und $n_{\mathrm{s}}=n_{1}=n_{2}$ die Brechungsindizes für den elektrischen Vektor einer linear polarisierten Lichtwelle parallel und senkrecht zur optischen Achse, die im vorliegenden Falle die Richtung des elektrischen Feldes hat, so werden die beiden Komponenten einer . monochromatischen Lichtwelle nach dem Durchlaufen der Dicke $l$ eines anisotropen Mediums eine Phasendifferenz von

$$
\delta=\frac{l}{\lambda} \cdot\left(n_{\mathrm{p}}-n_{\mathrm{s}}\right) 2 \pi=\frac{l}{\lambda} 2 \pi \Delta n
$$

( $\lambda=$ Lichtwellenlänge) haben. Je nachdem, ob $\delta>0$ oder $\delta<0$ ist, spricht man von positiver oder negativer Doppelbrechung.

2. Spezielle Theorie der elektrischen Doppelbrechung

Die folgenden Ausführungen stützen sich im wesentlichen auf die Arbeiten von Benort (1951, 1952) ${ }^{11,12}$, Stuart $(1939,1953)^{13,14}$ sowie von Peterlin und Stuart (1943, 1953) ${ }^{15,16}$. Darüber hinaus wurden auch die älteren theoretischen und z. T. auch methodisch grundlegenden Arbeiten von Debye (1913) ${ }^{17}$, Thummers (1914) ${ }^{18}$ und BorN (1918) ${ }^{19}$ herangezogen.

Für die Erscheinungen der Doppelbrechung sind nur die elektrischen Konstanten des Mediums maßgebend; der Einfluß der magnetischen Permeabilität kann vernachlässigt werden. Die Rolle der Felder besteht bei den hier zu behandelnden Lösungen darin, daß sie lediglich auf den Orientierungszustand der Teilchen wirken. Hierbei soll gelten, daß bei den verwendeten Feldern die elektrische Doppelbrechung nach dem K e r r - Gesetz verläuft:

$$
\Delta n=K n_{0} E^{2} \text {. }
$$

( $|\leftleftarrows|=E=$ elektrische Feldstärke, $K=$ K e r r - Konstante, $n_{0}=$ Brechzahl der Lösung bei $E=0$ ).

Betrachtet man eine TMV-Lösung als Lösung stäbchenförmiger Teilchen, die man als langgestreckte Rotationsellipsoide auffassen kann, so

15 A. Peterlin u. H. A. Stuart, Doppelbrechung, insbesondere künstliche Doppelbrechung, in: Hand- u. Jahrb. chem. Physik, Bd. 8/IB, S. 54-112, 1943.

16 A. Peterlin u. H. A. Stuart, Künstliche Doppelbrechung, in: Die Physik d. Hochpolymeren, Bd. II, S. 569-616, 1953.

17 P. Debye, Ber. dtsch. phys. Ges. 15, 777 [1913].

18 J. H. Thummers, Diss., Utrecht 1914.

19 M. Born, Ann. Physik 55, 177 [1918]. 
werden die Teilchenlängsachsen bei Abwesenheit eines orientierenden Feldes nach dem Boltz. $\mathrm{m}$ a n n schen Gleichverteilungsgesetz angeordnet sein. Die Lösung ist dann optisch isotrop, gleichgültig, ob die Teilchen Eigenanisotropie besitzen oder nicht. Ist dagegen ein elektrisches Feld vorhanden, so ist zur Ausrichtung der Teilchen deren elektrische Anisotropie notwendig, da nur dann auf die Teilchen ein Drehmoment ausgeübt werden kann. Diese Voraussetzung ist gegeben, wenn

1. die Teilchen selbst einen permanenten elektrischen Dipol darstellen oder

2. durch das äußere Feld ein elektrischer Dipol influenziert wird.

Wenn man so die Doppelbrechung als Folge der Orientierung stäbchenförmiger Teilchen (Makromoleküle) ansieht, wird man erwarten können, daß die Orientierung infolge der Zähigkeit des Lösungsmittels und der Teilchenträgheit dem orientierenden Feld verzögert folgt. Man nennt die Dauer der Verzögerung "Relaxationszeit" und bezeichnet sie allgemein mit $\tau$. Infolge dieser Relaxation sind zwei Effekte zu erwarten:

1. Die Intensität der Doppelbrechung fällt mit wachsenden Frequenzen des angelegten Wechselfeldes $a b$;

2. die Doppelbrechung ist gegenüber dem angelegten Wechselfeld phasenverschoben.

Die vorliegenden Untersuchungen beruhen ausschließlich auf Erscheinungen der zweiten Art. Die ebenfalls vorkommenden Intensitätsmessungen beruhen darauf, daß zwischen der Intensität der Doppelbrechung und der TMV-Konzentration Proportionalität besteht.

Die Relaxationszeit hängt mit der Rotationsdiffusions-Konstanten $D_{\mathrm{r}}$ durch die Beziehung

$$
\tau=\frac{1}{3 D_{\mathrm{r}}}
$$

zusammen. $\tau$ und damit auch $D_{\mathrm{r}}$ können als wichtige Elemente zur Bestimmung von Form und Größe der Makromoleküle herangezogen werden. Für Rotationsellipsoide haben Gans (1928) ${ }^{20}$ und Perrin (1934) ${ }^{21}$ unter der Annahme, daß sich die Rotation um eine Querachse, d. h. um eine Achse des größten Trägheitsmomentes, vollzieht, die $D_{\mathrm{r}}$ berechnet. Für den hier interessanten Fall eines Längen-DickenVerhältnisses $p>10$ gilt als Näherungsgleichung

$$
D_{\mathrm{r}}=\frac{k T}{2 \eta v} \cdot \frac{1}{p^{2}}(\ln 2 p-0,5)
$$

( $\eta=$ Viskositätskoeffizient der Lösung, $k=\mathrm{B}$ ol t z m a n n-Konstante, $T=$ absolute Temperatur, $v=$ Teilchenvolumen).

Man hat hiernach die Möglichkeit, $D_{\mathrm{r}}$ aus anderweitig ermittelten Molekülabmessungen (z. B. mit Hilfe des Elektronenmikroskopes) zu bestimmen.

Für den Zusammenhang zwischen elektrischem Feld und der Molekülorientierung sollen folgende Annahmen gelten:

a) Freie Drehbeweglichkeit der gelösten Teilchen;

b) die Lösung wird als weitgehend monodispers angesehen;

c) die Teilchen sollen starr und Rotationsellipsoide mit den Halbachsen $a_{1} / 2, a_{2} / 2, a_{3} / 2$ sein, wobei $a_{1}=a>a_{2}=a_{3}$ gelte;

d) geometrische, optische und elektrische Achsen sollen mit der Symmetrieachse zusammenfallen und

e) die allgemeinen Gesetze der B r o w n schen Molekularbewegung sollen anwendbar sein.

Die Richtungsverteilung der Molekülachsen $a$ sei durch die zeitabhängige Verteilungsfunktion

$$
f(\varphi, \vartheta, t)
$$

gegeben, die hier nicht näher untersucht werden soll ( $\varphi$ und $\vartheta$ sind Winkelkoordinaten auf der Einheitskugel). Ist kein orientierendes Feld vorhanden, so sind alle Richtungen gleich wahrscheinlich und die Verteilungsfunktion wird nach geschlossener Integration $1 /(4 \pi)$. Betrachtet man dagegen die Teilchenorientierung in einem elektrischen Feld, so kann man für die Verteilungsfunktion $f$ und den Orientierungs,strom“ $f \vec{\sigma}$ die Kontinuitätsgleichung

$$
\partial f / \partial t=\operatorname{div}(f \vec{\sigma})
$$

ansetzen, in der $\vec{\sigma}$ die Winkelgeschwindigkeit der Achsorientierung ist.

Für die folgenden Betrachtungen über die Doppelbrechung setzen wir zanächst voraus, daß die Amplitude des elektrischen Vektors der Lichtwelle im betrachteten Punkte klein ist im Vergleich zu der des angelegten elektrischen Wechselfeldes, das außerdem gegenüber der Lichtwellenfrequenz als konstant angesehen werden soll. Es kann dann von einem zusätzlichen Beitrag zur Polarisation abgesehen werden.

Ist das orientierende elektrische Feld konstant, dann ist die Relaxationszeit die Zeitspanne, die der

20 R. Gans, Ann. Physik 86, 628 [1928].

21 F. Perrin, J. Phys. Radium 5, 497 [1934], zitiert nach Peterlin und Stuart, 1953. 
Abfall des Ordnungsgrades oder der Doppelbrechung auf den $e$-ten Teil benötigt, wenn plötzlich das Feld entfernt wird. Bezeichnet man mit $\Delta n_{0}$ die maximale Doppelbrechung bei angelegtem Feld, also z. Z. $t=0$, so gehorcht $\Delta n$ dem üblichen Exponentialgesetz

$$
\Delta n=\Delta n_{0} e^{-t / \tau} .
$$

Setzt man in diese Gleichung für $\tau$ die Gl. (6) ein, so erhält man:

$$
\Delta n=\Delta n_{0} e^{-3 D_{\mathrm{r}} t} .
$$

Da bei Abschalten des Feldes der Ordnungsgrad nur durch die Wärmebewegung abgebaut wird, hat hier ein eventueller Dipolcharakter der Teilchen keinen Einfluß auf diese Gleichungen.

Hat das orientierende Feld die Form

$$
\mathfrak{E}=\mathfrak{E}_{0} e^{i \omega t},
$$

so werden die Anteile, die einmal durch einen influenzierten und zum anderen durch einen permanenten Dipol hervorgerufen werden, getrennt betrachtet werden müssen.

Bei der durch den influenzierten Dipol verursachten Komponente sind für die Teilchenorientierung die Richtungen parallel und antiparallel zum Feld gleichwertig. Die Doppelbrechung setzt sich hier aus einem konstanten Anteil $\Delta n_{0}$ zusammen, der von einem Wechselanteil überlagert wird. Jener stellt den zeitlichen Mittelwert dar, ist von der Zeit unabhängig und entspricht dem Wert, den man erhalten würde, wenn man an Stelle des Wechselfeldes ein konstantes Feld $\mathfrak{F}=\mathfrak{F}_{\text {eff }}$ anlegte. Der Verlauf von $\Delta n$ ist dann durch die folgende Gleichung

mit

$$
\Delta n=\Delta n_{0}\left[1 \pm \frac{\cos \left(2 \omega-\varphi_{1}\right)}{\sqrt{1+(\omega \tau)^{2}}}\right]
$$

$$
\operatorname{tg} \varphi_{1}=\omega \tau
$$

gegeben, die in Abb. 1 graphisch dargestellt ist. $\tau / 2$ ist die Relaxationszeit des Wechselanteiles gegenüber dem elektrischen Feld, da er gegenüber dem Feld mit der doppelten Frequenz schwingt.

Die Änderung der Doppelbrechung, die durch einen permanenten Dipol hervorgerufen wird, enthält dagegen keinen konstanten und von der Frequenz unabhängigen Anteil, da hier die Teilchenrichtungen parallel und antiparallel zum Feld nicht gleichwertig sind. Man erhält hier die Gleichung

$$
\Delta n=\Delta n_{0}\left[\frac{1}{1+\left(\omega \tau^{*}\right)^{2}} \pm \frac{\cos \left(2 \omega t-\varphi_{2}\right)}{\sqrt{1+\left(\omega \tau^{*}\right)^{2} \cdot \sqrt{1+(\omega \tau)^{2}}}}\right]
$$

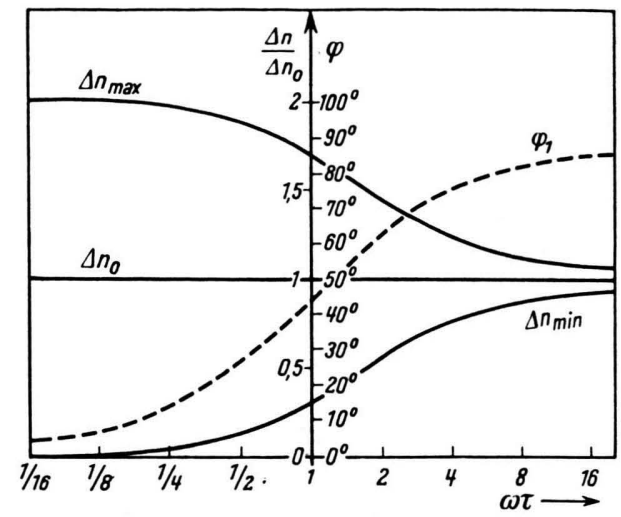

Abb. 1. Größe und Phasenverschiebung der Doppelbrechung durch einen influenzierten Dipol in Abhängigkeit von der Frequenz.

mit

$$
\operatorname{tg} \varphi_{2}=\frac{5 \omega \tau}{2-3(\omega \tau)^{2}}
$$

und der dem Mittelwert entsprechenden Relaxationszeit

$$
\tau^{*}=\frac{3}{2} \tau=\frac{1}{2 D_{\mathrm{r}}} .
$$

Die graphische Darstellung zeigt die Abb. 2.

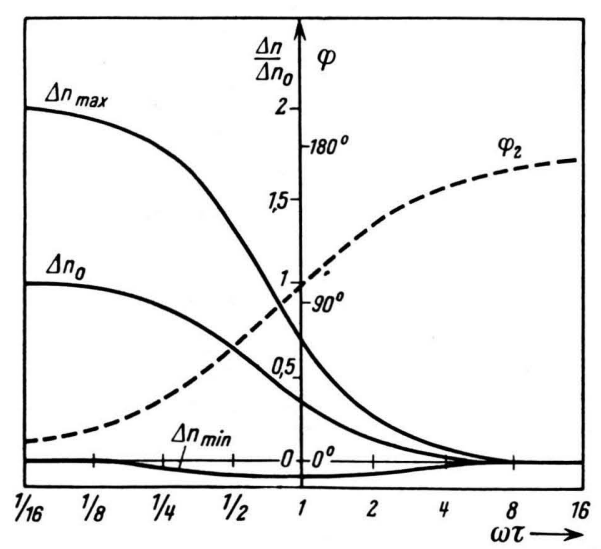

Abb. 2. Größe und Phasenverschiebung der Doppelbrechung durch einen permanenten Dipol in Abhängigkeit von der Frequenz.

Experimentell konnte für die TMV-Lösungen bisher von Benoit (1952) ${ }^{12}$ sowie O'Konski und HaltNER $(1957)^{8}$ lediglich die Gl. (12) bestätigt werden.

\section{Spezielle Theorie zur Versuchsdurchführung}

Für den Aufbau der Anlage ist die Lösung des folgenden Problems ausschlaggebend gewesen: Bei 
einem bestimmten, die gelösten Teilchen orientierenden Feld der Form

$$
E=a e^{i \omega t}
$$

ist die Abhängigkeit der Doppelbrechung vom Feld zu bestimmen, insbesondere deren Relaxation. Um einen allzu großen apparativen Aufbau zu umgehen, wurde ein sinusförmiges Wechselfeld mit konstanter Frequenz und Amplitude verwendet.

Wegen der raschen Folge der DoppelbrechungsÄnderungen ist es nicht möglich, mit Hilfe der üblichen Kompensatoren den Gangunterschied $\delta$ direkt zu messen, sondern es mußte der Weg über Intensitätsmessungen beschritten werden. Man erhält aber aus zwei superponierten und phasenverschobenen Wellenzügen die Intensität, wenn man deren zeitlichen Mittelwert des Quadrates bildet. Deshalb ist der Zusammenhang zwischen Intensität und Gangunterschied $\delta$ nicht mehr von der einfachen Form der Gl. (4). Hierauf soll jedoch nicht näher eingegangen werden, weil es bei der Ermittlung der Relaxations-Erscheinungen nicht auf die Absolutwerte der Doppelbrechung ankommt. Es handelt sich im folgenden hierbei immer um Relativwerte, und nur in diesem Sinne ist auch weiterhin der Ausdruck „Amplitude der Doppelbrechung “ zu verstehen.

Da sich die Frequenzen der Doppelbrechung und des Feldes wie $2: 1$ verhalten, wird man auf die hierbei üblichen Li s s a j o u s - Figuren zurückgreifen, die im Idealfalle durch die Angabe dreier Kurvenpunkte eindeutig bestimmt sind. Da aber Voruntersuchungen gezeigt haben, daß die Doppelbrechung sich in ihrem Verlauf verändert und die gemessenen Intensitäten im allgemeinen eine nichtharmonische Schwingung darstellten, die durch eine F o u ri er - Entwicklung bis zum Glied für die erste Oberwelle hinreichend genau wiedergegeben wurden, hat man die beiden senkrecht aufeinander stehenden Schwingungen:

$$
x=a \cos \omega t,
$$

$y=b \cos (2 \omega t-\varphi)+c \cos (4 \omega t-2 \varphi)+d$,

wobei $x$ dem elektrischen Feld und $y$ der Doppelbrechung entspricht. Diese beiden Gleichungen sind dann die Parameterdarstellung der resultierenden Kurve zweiten Grades, die qualitativ für einen speziellen Fall auf der Abb. 3 dargestellt ist.

Zur Bestimmung der vier Unbekannten, der Phasenverschiebung $\varphi$ und der Koeffizienten $b, c, d$ sind mindestens vier Gleichungen notwendig, die man durch Ausmessen charakteristischer Kurvenpunkte

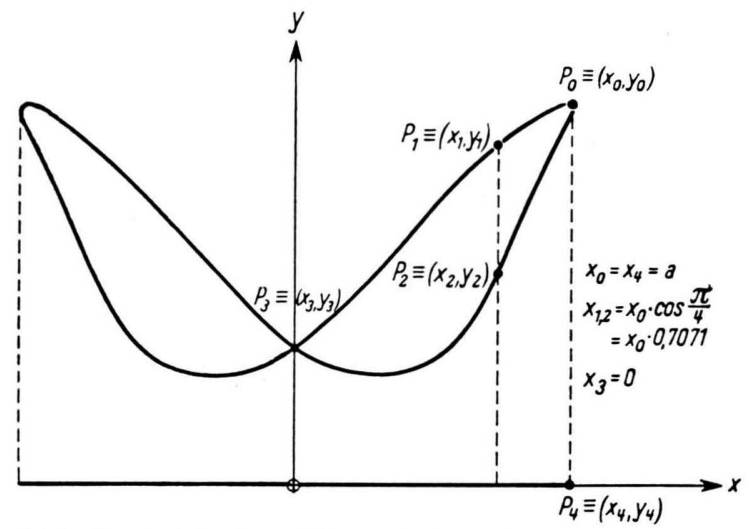

Abb. 3. Qualitativer Verlauf der Kurve $x=a \cdot \cos \omega t$, $y=b \cdot \cos (2 \omega t-\varphi)+c \cdot \cos (4 \omega t-2 \varphi)+d$ für einen speziellen Fall.

$P_{v}(v=0,1,2,3)$ erhalten kann. Wird ider Einfachheit halber $\omega t=\alpha$ gesetzt, so erhält man für bestimmte $\alpha$-Werte ganz bestimmte Punkte $P$ der Kurve. Damit sich die Gleichungen rechnerisch einfach gestalten ließen, wurden folgende $\alpha$-Werte gewählt, die vier Kurvenpunkten entsprechen:

$$
\begin{array}{ll}
P_{0}: & \alpha_{0}=0, \\
P_{1}: & a_{1}=+\pi / 4, \\
P_{2}: & \alpha_{2}=-\pi / 4, \\
P_{3}: & \alpha_{3}= \pm \pi / 2 .
\end{array}
$$

Setzt man diese Werte in die Gl. (15') ein, so erhält man vier Gleichungen für vier Unbekannte, so daß deren Bestimmung möglich wird. Ist der Koeffizient der ersten Oberschwingung $(c)$ vernachlässigbar klein, so ist die Konstante $b$ gleichzeitig die Amplitude der Doppelbrechung. Kann $c$ nicht vernachlässigt werden, so gilt

und

$$
\Delta n_{\max }=b-c
$$

$$
\Delta n_{\min }=b+c,
$$

was auch experimentell bestätigt werden konnte.

Die Phasenverschiebung läßt sich aus dem Tangens ermitteln:

$$
\operatorname{tg} \varphi=\frac{y_{1}-y_{2}}{y_{0}-y_{3}} .
$$

Mit ihr läßt sich die Relaxationszeit $\tau$ nach der Gleichung

$$
\tau=\frac{\varphi}{360} \cdot \frac{t}{2}
$$

berechnen $(t=$ Dauer einer Schwingungsperiode des elektrischen Wechselfeldes). Um die Rotationsdiffusions-Konstante $D_{\mathrm{r}}$ zu erhalten, setzt man $\tau$ in die Gl. (6) ein und löst nach $D_{\mathrm{r}}$ auf. 


\section{Experimenteller Teil}

1. Versuchs a $\mathrm{f} b \mathrm{au}$

Es galt eine Versuchsanordnung herzustellen, mit der einmal der Amplitudenverlauf der Doppelbrechung und zum anderen deren Phasenverschiebung gegenüber dem orientierenden Feld bestimmt werden konnten. Die Abb. 4 zeigt schematisch den Aufbau des Versuches. an 220 Volt Wechselspannung, die dem Lichtnetz ent nommen wurde. Spannungsschwankungen traten hierbei nur vereinzelt auf und betrugen nie mehr als 3 Prozent. Die gleiche Spannung diente auch zur Horizontalauslenkung des Kathodenstrahles.

Die Erwärmung der Lösungen erfolgte im Temperiertrog, der von einem Höppler-Thermostaten aus gesteuert werden konnte.

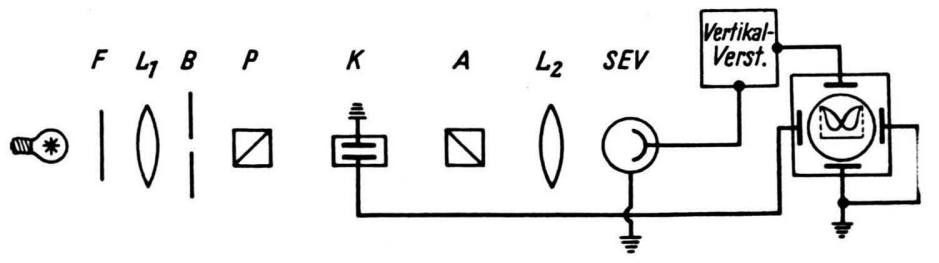

\begin{abstract}
Abb. 4. Schematische Darstellung des Versuchsaufbaues $\left(\mathrm{F}=\right.$ Blaufilter, $\mathrm{L}_{1,2}=\mathrm{Lin}$ sen, $\mathbf{B}=$ Blende, $\mathbf{P}=$ Polarisator, $\mathrm{K}=$ $\mathrm{K}$ e r r - Zelle, $\mathrm{A}=$ Analysator, $\mathrm{SEV}=$ Sekundärelektronen-Vervielfacher).
\end{abstract}

a) Lichtquelle: Als Lichtquelle diente eine übliche Mikroskopierleuchte, die aus einem 6-Volt-Akkumulator gespeist wurde. Die Stromkonstanz war für die Dauer eines Versuches gegeben. Mit einer rotierenden Lochscheibe konnte der Lichstrahl in beliebiger Folge unterbrochen werden, was ein gleichzeitiges Registrieren der Kurve und der zugehörigen Grundlinie $y=0$ ermöglichte. Die Unterbrecherfrequenz war $0,6-1 \mathrm{kHz}$. Um die Helligkeit der Lichtquelle auf ein für die Versuche günstiges $\mathrm{Ma} \beta$ zu vermindern, wurde ein Blaufilter $F$ aus Kobaltglas in den Strahlengang geschaltet. Dadurch konnte auch die Wärmestrahlung wesentlich unterdrückt werden.

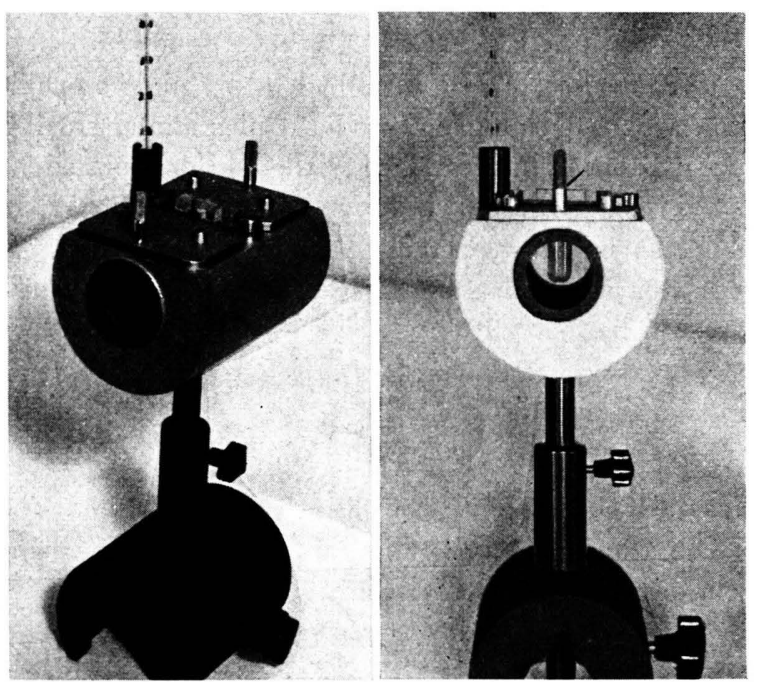

Abb. 5. Anordnung der K e r r-Küvette im Temperiertrog.

b) Küvettenteil: Zur Aufnahme der zu untersuchenden TMV-Lösungen diente eine Zeiss'sche Meßküvette mit $1 \mathrm{~cm}$ Schichtdicke, wie sie bei den üblichen $P$ ulf ri c h-Photometern verwendet wird. Die Lage und die Halter dieser Küvette mit den beiden aus V 2 A-Stahl angefertigten Elektroden im Temperiertrog gehen aus der Abb. 5 hervor.

Während der Versuche lagen die beiden Elektroden
Als Polarisatoren dienten handelsübliche „Herotare“ vom VEB Carl Zeiss Jena.

c) Registrierteil: Durch die Linse $\mathrm{L}_{2}$ wurde die Mitte der Ke r r-Küvette auf die Photokathode des Sekundärelektronen-Vervielfachers abgebildet und dessen Anodenstrom an den Eingang vom Vertikalverstärker des Elektronenstrahl-Oszillographen sowie über einen Ableitwiderstand an Erde gelegt. Die Abb. 6 zeigt das Schaltbild für den Vervielfacher (SEV Typ M 12 S). Die an der Potentiometerkette liegende Gesamtspannung von $800-1100$ Volt stammt von in Reihe geschalteten Anodenbatterien. Der Querstrom des Spannungsteilers war bei dieser Anordnung mindestens um eine Zehnerpotenz größer als der maximal zulässige Anodenstrom des Vervielfachers für Gleichlicht, was für eine gute Arbeitsweise zu empfehlen ist. Die variablen Widerstände $R_{1}, R_{12}$ und $R_{13}$ wurden für den verwendeten Vervielfacher auf die optimalen Werte eingestellt und nicht mehr verändert. Im allgemeinen ließen sich die Messungen bei einer Gesamtspannung von 800 Volt durchführen. Die Konstanz der Intensitätsmessungen war über eine Dauer von 5 Stdn. nur mit einem Fehler von weniger als $0,5 \%$ behaftet.

\section{Herstellung der TMV-Lösung}

Allgemein sind zur Isolierung von Viren aus Pflanzensäften drei Verfahren üblich:

1. Isolierung mit Hilfe der Ultrazentrifuge,

2. Trennung durch Elektrophorese, und

3. Reinigung durch Fällung.

Darüberhinaus sind auch Kombinationen dieser Methoden möglich, deren Zweckmäßigkeit sich nach der jeweiligen Aufgabenstellung richten wird.

Die in dieser Arbeit verwendeten TMV-Lösungen wurden vorwiegend auf dem Wege der Fällung hergestellt.

Von den zahlreichen Stämmen des TMV wurde der Dahlemer "Gelbstamm" verwendet, der Nicotiana tabacum systemisch infiziert und auf $N$. sylvestris Lokalläsionen verursacht.

Zur Herstellung der Rohsäfte habe ich 25 systemisch infizierte Tabakpflanzen der Sorte "Samsun" $(3-5$ Wochen nach der Infektion) mit der vierfachen Menge 

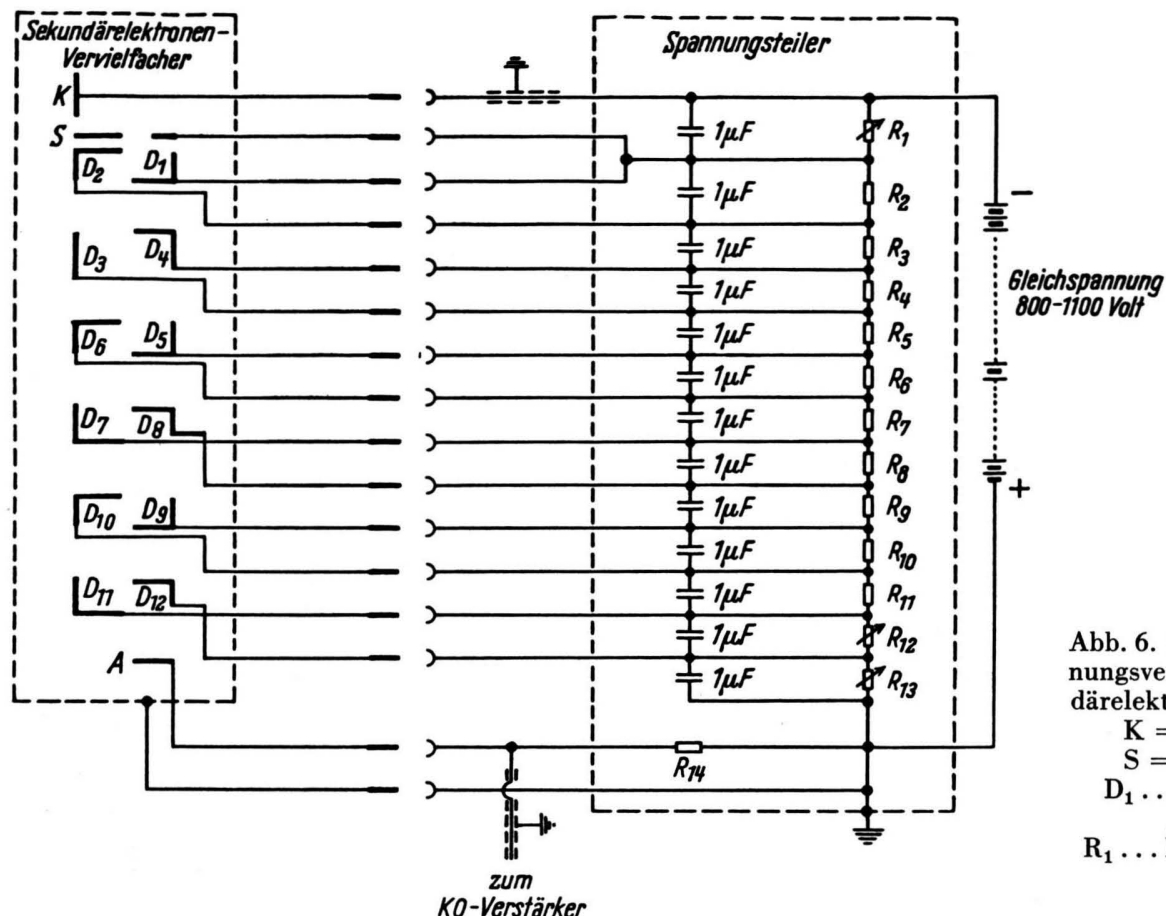

Abb. 6. Schaltung zur Spannungsversorgung des Sekundärelektronen-Vervielfachers.

$\mathrm{K}=$ Photokathode;

$\mathrm{S}=$ Hilfselektrode;

$D_{1} \ldots D_{12}=$ Dynoden; $\mathrm{A}=$ Anode

$R_{1} \ldots R_{14}=$ Widerstände.

dest. Wassers homogenisiert. Nach einer Vorreinigung von groben Teilchen durch niedertouriges Zentrifugieren wurde das Eiweiß mit Ammoniumsulfat ausgefällt und von den übrigen Pflanzenstoffen isoliert. Dieser Vorgang mußte 4-5-mal wiederholt werden. Die Trennung des Virusproteins vom Pflanzeneiweiß erfolgte durch mehrmalige isoelektrische Fällung, beim TMV also bei einem $p_{\mathrm{H}}$-Wert von $3,3-3,5$. Das Entsalzen der Lösungen konnte durch mehrstdg. Elektrodialyse erreicht werden.

Die Kontrolle auf Reinheit der TMV-Lösungen sowie alle anderen elektronenmikroskopischen Untersuchungen wurden mit dem Elektronenmikroskop „Elmi D 2“ vom VEB Carl Zeiss Jena durchgeführt.

Die gravimetrisch ermittelte TMV-Konzentration ergab $(0,71 \pm 0,07) \mathrm{mg} / \mathrm{ml}$ der Lösung, wenn man an nimmt, daß keine Verunreinigungen mehr enthalten sind. Bei dieser Konzentration kann die Lösung als „ideal“ angesehen werden, da hier noch keine Behinderung der Teilchen durch Nachbarteilchen erfolgt, also freie Drehbeweglichkeit gegeben ist.

\section{Durchführung der Messungen und Meßergebnisse}

a) Beschreibung des MeBvorganges: Nach dem Füllen der Ke r r-Küvette mit der TMV-Lösung wurde die Elektrodenspannung angelegt und unmittelbar danach mit der ersten Aufnahme und der Zeitmessung begonnen. Der Temperatur-Zeit-Verlauf ist aus dem folgenden Diagramm (Abb. 7) ersichtlich und war stets reproduzierbar.

Zur Auswertung der Lis s a j o u s - Figuren wurden die Leuchtschirmbilder mit einer Kleinbildkamera auf
AGFA-Dokumentenfilm photographiert und die Negative unter dem „Dokumator-Lesegerät“ ausgemessen. Die Berechnung von Amplitude und Phasenwinkel erfolgte unter Anwendung der im theoretischen Teil abgeleiteten Gleichungen.

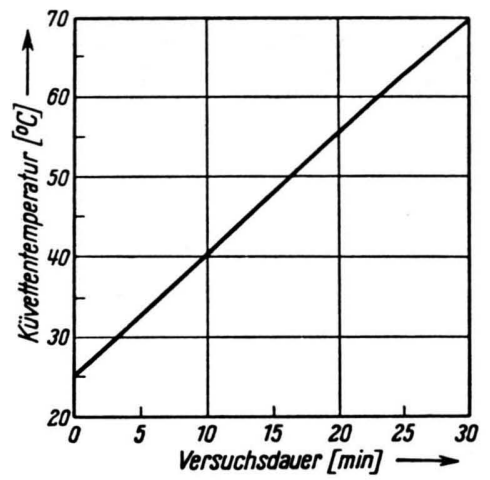

Abb. 7. Temperatur der $\mathrm{K}$ e $\mathrm{r} \mathrm{r}$ - Zelle in Abhängigkeit von der Versuchsdauer.

Nach dem Verschwinden der Doppelbrechung waren die Versuche beendet, und für die elektronenmikroskopischen Untersuchungen wurden der Küvette entsprechende Proben entnommen.

b) Versuchsergebnisse: Zunächst wird der Fall der kontinuierlichen Erwärmung betrachtet. Die aus der Ausmessung der Lis s a jou s-Figuren sich ergebenden Werte für die Phasenverschiebung $\varphi$, die Relaxationszeit $\tau$, die Rotationsdiffusions-Konstante $D_{\mathrm{r}}$ einerseits und die Amplitude der Doppelbrechung anderer- 


\begin{tabular}{|c|c|c|c|c|}
\hline $\begin{array}{c}\text { Temperatur } \\
\frac{T}{-} \\
{\left[{ }^{\circ} \mathrm{C}\right]}\end{array}$ & $\begin{array}{c}\text { Phasenverschiebung } \\
\varphi \\
\text { Gl. }(17) \\
{\left[{ }^{\circ}\right]}\end{array}$ & $\begin{array}{c}\text { Relaxationszeit } \\
\tau \\
\text { Gl. }(18) \\
\text { [sec. } 10^{-6} \text { ] }\end{array}$ & $\begin{array}{c}\text { Rotationsdiffusions- } \\
\text { Konstante } \\
D_{\mathrm{r}} \\
\text { Gl. }(7) \\
{\left[\text { sec. }^{-1}\right]}\end{array}$ & $\begin{array}{c}\text { Amplitude der } \\
\text { Doppelbrechung } \\
\Delta n \\
\text { Gln. (16) und }\left(16^{\prime}\right) \\
\text { willkürliche Einheit }\end{array}$ \\
\hline $\begin{array}{l}25,0 \\
30,0 \\
34,5 \\
37,5 \\
40,0 \\
44,0 \\
46,0 \\
50,0 \\
53,0 \\
55,5 \\
57,5 \\
60,5 \\
63,0 \\
66,0 \\
68,5 \\
70,0 \\
71,5 \\
72,0\end{array}$ & $\begin{array}{l}27,4 \pm 0,2 \\
27,3 \pm 0,2 \\
27,0 \pm 0,1 \\
26,7 \pm 0,2 \\
26,7 \pm 0,2 \\
27,8 \pm 0,4 \\
28,1 \pm 0,3 \\
28,2 \pm 0,2 \\
28,8 \pm 0,4 \\
29,7 \pm 0,3 \\
30,3 \pm 0,5 \\
31,5 \pm 0,6 \\
32,6 \pm 0,4 \\
33,3 \pm 0,4 \\
31,6 \pm 0,6 \\
19,1 \pm 0,5 \\
23,3 \pm 0,5 \\
19,4 \pm 0,8\end{array}$ & $\begin{array}{l}762 \pm 5 \\
760 \pm 5 \\
751 \pm 3 \\
743 \pm 3 \\
743 \pm 5 \\
773 \pm 12 \\
782 \pm 8 \\
785 \pm 5 \\
801 \pm 12 \\
826 \pm 9 \\
843 \pm 14 \\
877 \pm 16 \\
908 \pm 10 \\
944 \pm 11 \\
880 \pm 16 \\
810 \pm 20 \\
648 \pm 14 \\
539 \pm 23\end{array}$ & $\begin{array}{l}438 \pm 3 \\
439 \pm 3 \\
444 \pm 1 \\
448 \pm 3 \\
448 \pm 3 \\
432 \pm 7 \\
426 \pm 4 \\
425 \pm 3 \\
417 \pm 7 \\
404 \pm 5 \\
395 \pm 6 \\
380 \pm 6 \\
367 \pm 4 \\
353 \pm 4 \\
379 \pm 7 \\
412 \pm 10 \\
514 \pm 10 \\
618 \pm 25\end{array}$ & $\begin{array}{r}21,1 \pm 0,6 \\
28,5 \pm 1,3 \\
37,6 \pm 3,3 \\
43,4 \pm 2,6 \\
47,7 \pm 0,2 \\
51,8 \pm 1,4 \\
53,7 \pm 1,1 \\
56,7 \pm 1,1 \\
58,7 \pm 1,2 \\
61,5 \pm 1,0 \\
62,4 \pm 0,4 \\
61,6 \pm 0,4 \\
60,0 \pm 0,2 \\
58,8 \pm 0,4 \\
50,0 \pm 3,3 \\
26,5 \pm 5,5 \\
11,4 \pm 1,8 \\
2,8 \pm 1,0\end{array}$ \\
\hline
\end{tabular}

Tab. 1. Phasenverschiebung $\varphi$, Relaxationszeit $\tau$, Rotationsdiffusions-Konstante $D_{\mathrm{r}}$ und Amplitude der Doppelbrechung in Abhängigkeit von der Erwärmung.

seits sind bei den einzelnen Temperaturstufen aus der Tab. 1 ersichtlich. Die vorliegenden Zahlen sind die arithmetischen Mittelwerte aus je 5 Liss a jous Figuren, wie sie zu den einzelnen Temperaturpunkten gehören.

Zur graphischen Darstellung dieser Ergebnisse wurden lediglich die Amplitude und die Phasenverschiebung herangezogen, da sich die anderen Werte von $\varphi$ nur durch konstante Faktoren unterscheiden und deshalb gleiche Kurvenverläufe zeigen würden. Das Ergebnis ist in Abb. 8 dargestellt.

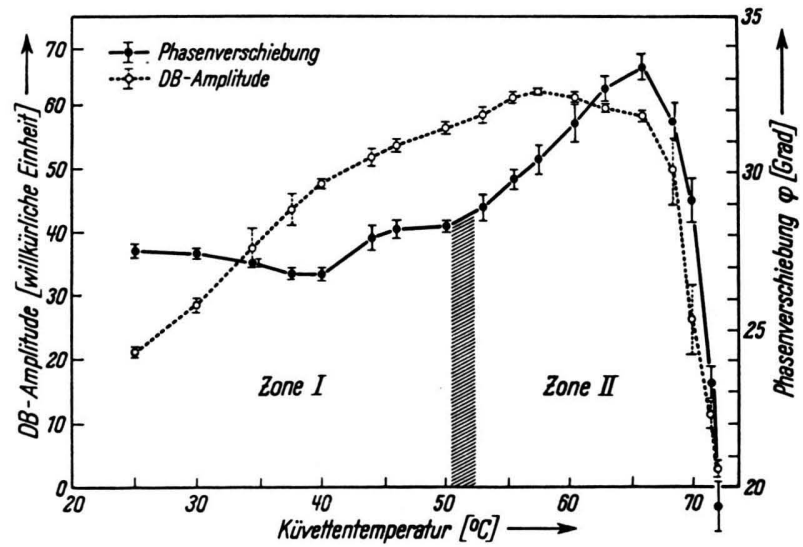

Abb. 8. Amplitude und Phasenverschiebung der Doppelbrechung in Abhängigkeit von der Küvettentemperatur.

22 S. W. Fox u. J. F. Foster, Introduction to protein chemistry. S. 304-326, New York 1957.

23 T. Fukushi u. E. Shikata, J. Fac. Agric. Hokkaido Imp. Univ. 50, 74 [1955].
Man sieht aus dem Verlauf der Kurven, daß beim kontinuierlichen Erwärmen zunächst kein wesentlicher Einfluß auf die Phasenverschiebung ausgeübt wird. Dieser Bereich, der bis $(51,5 \pm 1,0){ }^{\circ} \mathrm{C}$ reicht, soll als Zone I bezeichnet werden. Der hier zu Beginn auftretende schwache Abfall von $\varphi$ dürfte auf die Verminderung der Viskosität des Wassers zurückzuführen sein. Der geringe Anstieg, bei etwa $40^{\circ} \mathrm{C}$ beginnend, kann als Folge einer geringen Teilchenstreckung angesehen werden, die zunächst der völligen Entfaltung der Polypeptidketten und der Koagulation des Proteins vorausgeht. (Fox und Foster, 1957) ${ }^{22}$. Die sich anschließende Zone II ist dadurch charakterisiert, daß in ihr die Phasenverschiebung anfangs erheblich zunimmt. Es müssen demnach in diesem Bereich Faktoren auftreten, die die Drehbeweglichkeit der Teilchen einschränken. Hierfür kann man zwei Ursachen annehmen, da eine Erhöhung des Reibungswiderstandes gewiß nicht in Betracht kommt :

1. Verminderung des Dipolmomentes und

2. Vergrößerung des Trägheitsmomentes.

Eine Entscheidung über den unter Punkt 1 genannten Einfluß kann nach dem augenblicklichen Stand der Untersuchungen nicht getroffen werden. Für die unter Punkt 2 genannte Vergrößerung des Trägheitsmomentes spricht zunächst schon die Tatsache, daß nach den elektronenmikroskopischen Untersuchungen die Koagulation des TMV-Proteins vorwiegend an den Enden der Stäbchen beginnt, wobei durch Masseverlagerung eine erhebliche Vergrößerung des Trägheitsmomentes verbunden sein könnte. Ferner legt ein Vergleich der elektronenmikroskopischen Aufnahmen die Vermutung nahe, daß neben dem Koagulationsprozeß auch in gehäufter Zahl Längsaggregationen auftreten, was nach den Ergebnissen von Fukushi und Shikata $(1955)^{23}$ auch zu erwarten 
ist. Diese Autoren stellten bei elektronenmikroskopischen Längsvermessungen fest, daß bei den TMV-Lösungen, die $10 \mathrm{Min}$. auf $60^{\circ} \mathrm{C}$ erhitzt worden sind, die Längsaggregationen auf Kosten der Bruchstücke zunahmen. Bei höheren Temperaturen oder längeren Zeiten trat der gegenteilige Effekt auf.

Der schnelle Abfall von $\varphi$ bei über $66^{\circ} \mathrm{C}$ hängt mit dem Verlust des K e r r-Effektes der Lösung zusammen. Dieser tritt ein, wenn der größte Teil des Proteins koaguliert ist und die Zahl der noch intakten Teilchen nicht mehr ausreicht, um eine meßbare Doppelbrechung zu erzeugen. Bei der Protein-Denaturierung wird der Ordnungszustand in der Polypeptidkette durch die Sprengung der innermolekularen Wasserstoffbrücken zunächst zerstört; da diese sich aber teilweise und vor allem regellos wieder ausbilden, kommt es zur Koagulation des Eiweißes zu kugelförmigen Teilchen, die keinen K e r r-Effekt mehr verursachen. Während der Temperaturbereich der Zone I für verschiedene Lösungen annähernd konstant bleibt, scheint der Bereich der Zone II lösungsabhängig, insbesondere im Hinblick auf die Konzentration, zu sein.

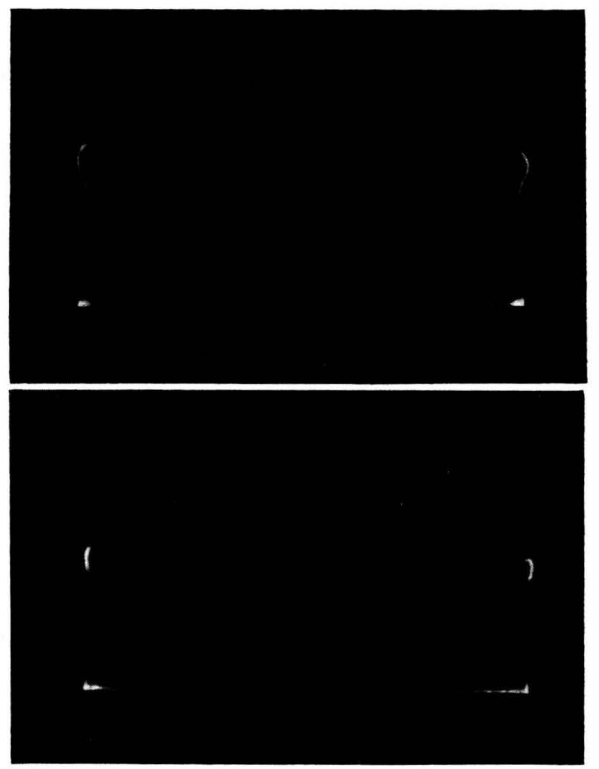

Abb. 9. Lis s a j o u s - Figuren; oben: bei Versuchsbeginn, unten: kurz vor dem Versuchsende.

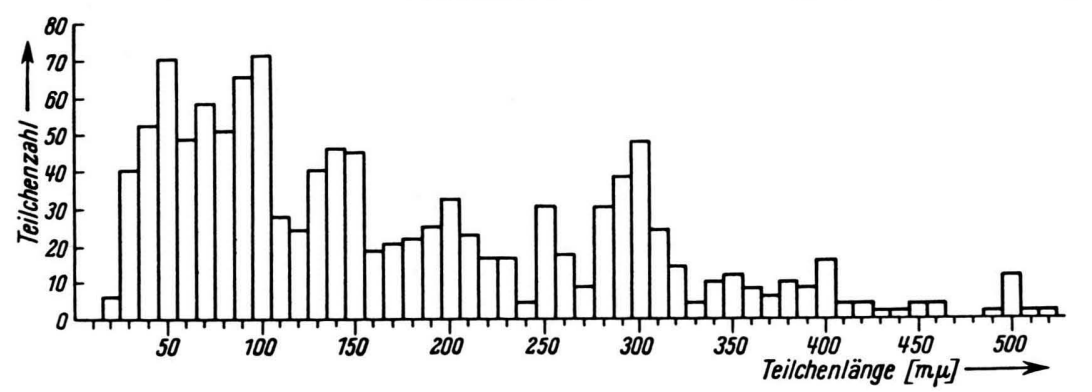

Abb. 12. Längenverteilung der TMV-Stäbchen in der Ausgangslösung.
Eine weitere Untersuchung galt dem Schwingungsverlauf der Doppelbrechung. Während zu Beginn der Versuche die Lis a jo u s - Figuren auf eine harmonische Schwingung der Doppelbrechung schließen ließen, traten mit zunehmender Erwärmung Veränderungen auf, die auf das Vorhandensein einer Oberschwingung hindeuteten. Ein Vergleich der Kurven von Abb. 9 macht dies deutlich.

c) Elektronenmikroskopische Untersuchungen: Ziel der elektronenmikroskopischen Untersuchungen war es, u. a. die morphologischen Veränderungen an den denaturierten TMV-Teilchen der Ausgangslösung festzustellen. Ein Vergleich der Partikeln, wie sie unmittelbar vor und nach dem Versuch vorliegen (Abb. 10 und $11^{*}$ ), zeigt Unterschiede. Man findet deutlich das zu kugelförmigen Teilchen denaturierte Protein, was mit einer erheblichen Abnahme an morphologisch intakten TMV. Stäbchen verbunden ist.

Ein weiterer Zweck dieser Untersuchung war es, die Rotationsdiffusions-Konstante $D_{\mathrm{r}}$ aus der Teilchenvermessung nach der Gl. (7) zu berechnen. Die hierzu notwendige genaue Verteilung der Teilchenlängen in der Ausgangslösung ist auf der Abb. 12 in der üblichen Diagrammform dargestellt. Die Stufenbreite beträgt $10 \mathrm{~m} \mu$. Das Abweichen des Hauptmaximums bei $100 \mathrm{~m} \mu$ von der „in vivo“ ermittelten Normallänge von $300 \mathrm{~m} \mu$ kann auf die Präparation zur Reindarstellung zurückgeführt werden. Die übrigen Nebenmaxima bei 50,140 , $200,250 \mathrm{~m} \mu$ usw. entsprechen ganzzahligen Vielfachen von $1 / 6$ der Normallänge; dies stimmt mit dem von Schramm und Wiedemann (1951) ${ }^{24}$ gefundenen Ergebnis überein, wonach das TMV bevorzugte Bruchstellen aufweist, die die Normallänge in $\mathbf{1} / \mathbf{6}$-Einheiten teilen.

Zur Phasenverschiebung einer solchen Lösung werden die Teilchen aller vorkommenden Längen einen entsprechenden Beitrag liefern. Man muß daher zur Berechnung von $D_{\mathrm{r}}$ nach der $\mathrm{Gl}$. (7) statt der Teilchenlänge $a$ die Schwerpunktlänge $a_{\mathrm{s}}$ zugrundelegen. Werden die einzelnen Längenklassen mit $x_{i}(i=1,2, \ldots, k ; k=$ Anzahl der Klassen) und deren zugehörige Teilchenzahl mit $z_{i}$ bezeichnet, so ergibt sich die Schwerpunktlänge $\mathrm{zu}$

$$
a_{\mathrm{s}}=\frac{\sum_{1}^{k} x_{i} z_{i}}{N},
$$

wenn $N$ die Gesamtzahl der ausgemessenen Teilchen ist.

\footnotetext{
* Abb. 10 u. 11 auf Tafel S. 152 b.
}

24 G. Schramm u. M. Wiedemann, Z. Naturforschg. 6 b, 379 [1951]. 


\begin{tabular}{|c|c|c|}
\hline$D_{\mathrm{r}}$-Wert $\left(\mathrm{sec}^{-1}\right)$ & Autor & Methode \\
\hline $\begin{array}{l}280 \pm 28 \\
303 \pm 60 \\
550 \pm 55 \\
440 \\
450 \\
333 \pm 13 \\
438 \pm 3 \\
404 \pm 50\end{array}$ & 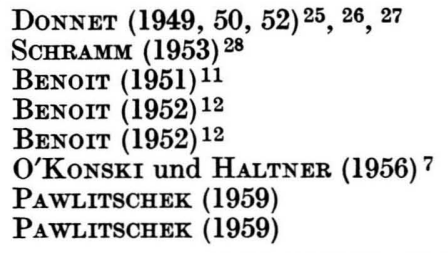 & $\begin{array}{l}\text { Strömungsdoppelbrechung } \\
\text { berechnet } \\
\text { elektrooptisch * } \\
\text { elektrooptisch } \\
\text { elektrooptisch * } \\
\text { elektrooptisch * } \\
\text { elektrooptisch } \\
\text { nach Gl. }\left(7^{\prime \prime}\right)\end{array}$ \\
\hline
\end{tabular}

Tab. 2. Vergleich einiger Literaturwerte für die Rotationsdiffusions-Konstante $D_{\mathrm{r}}$. ${ }^{*}$ Bei diesen Versuchen waren die elektrischen Felder Impulsfelder; in allen übrigen Fällen handelte es sich um sinusförmige Wechselfelder.

$a_{\mathrm{s}}$ ist der Mittelwert aus drei Bestimmungen, die unabhängig von drei Personen durch die Vermessung von etwa je 1000 Teilchen durchgeführt worden sind. Sein Wert war (151 \pm 5$) \mathrm{m} \mu$, wobei $\pm 5 \mathrm{~m} \mu$ der mittlere absolute Fehler ist. Aus dieser Schwerpunktlänge und der TMV-Dicke von $2 r=(15 \pm 1) \mathrm{m} \mu$ berechnet sich das Längen-Dicken-Verhältnis $p$ zu $(10,7 \pm 1,6)$. Setzt man diese Werte und für die Viskosität des Wassers bei $25^{\circ} \mathrm{C}=0,00894$ dyn. sec. $\mathrm{cm}^{-2}$ in die Gl. (7) ein, so erhält man die Rotationsdiffusions-Konstante $D_{\mathrm{r}}$.

Der Vergleich der beiden nach verschiedenen Methoden ermittelten Werte für die $D_{\mathrm{r}}$ zeigt befriedigende Übereinstimmung:

$$
\begin{aligned}
& D_{\mathrm{r}}=(404 \pm 50) \text { sec. }^{-1} \quad \text { [nach Gl. (7)] } \\
& D_{\mathrm{r}}=(438 \pm 3) \text { sec. }^{-1} \quad \text { (elektrooptisch) }
\end{aligned}
$$

\section{Diskussion}

Es ist bereits in der Einleitung erwähnt worden, daß das Ziel dieser Arbeit darin bestand, die Grundlagen für ein künftiges umfassenderes Arbeitsgebiet zu schaffen, das sich auf die Beobachtung von Denaturierungsvorgängen bei Pflanzenviren in wäßriger Lösung mit Hilfe des K e r r - Effektes erstreckt.

Die Kurve der Abb. 8 zeigt einen deutlichen $\mathrm{Zu}$ sammenhang der Phasenverschiebung und Amplitude der Doppelbrechung mit einer Erwärmung der Viruslösung, die bis zur Denaturierung des TMVProteins reicht. Eine eingehende Diskussion der einzelnen Kurvenabschnitte ist bereits an entsprechender Stelle gegeben worden.

Die in dieser Arbeit ermittelten Rotationsdiffusions-Konstanten bei Zimmertemperatur $\left(25^{\circ} \mathrm{C}\right) D_{\mathrm{r}}$ sollen hier mit den Werten, die andere Autoren mit

25 J. B. Donnet, C. R. hebd. Séances Acad. Sci. 229, 189 [1949], zitiert nach Peterlin und Stuart, 1953.

26 J. B. Donnet, C. R. hebd. Séances Acad. Sci. 47, 698 [1950], zitiert nach Peterlin und Stuart, 1953. z. T. anderen Methoden gefunden haben, verglichen werden (Tab. 2) :

Die Übereinstimmung der Werte ist befriedigend, wenn man bedenkt, daß der Aggregationszustand und die Konzentration nicht ohne Einfluß auf die Phasenverschiebung sind. Außerdem lag das Hauptgewicht der Fragestellung auf dem Zusammenhang der relativen Phasenverschiebung, also des Kurvenverlaufes, mit den Denaturierungsvorgängen,

Als neuer Befund kann die Feststellung gewertet werden, daß die Schwingung der Doppelbrechung, im Gegensatz zum sie erzeugenden sinusförmigen Wechselfeld, im allgemeinen nichtharmonisch ist. Der Grund hierfür könnte in der bisher verneinten Existenz eines permanenten Dipolanteiles der Doppelbrechung gesehen werden. Wie dann allerdings die Tatsache zu bewerten ist, daß sich diese unharmonische Schwingung während der Versuche ändert, bleibt ungeklärt. Eine weitere Deutung wäre die folgende: Das TMV-Molekül ist am isoelektrischen Punkt neutral, d. h. der Dissoziationsgrad der sauren Gruppen ist gleich dem der basischen Gruppen. Um die Lösungen salzfrei zu bekommen, wurden sie gegen zweifach dest. Wasser elektrisch dialysiert. Sie hatten damit einen $\mathrm{p}_{\mathrm{H}}$-Wert, der um den Neutralpunkt lag. Hierdurch wurde der Dissoziationsgrad der positiven $\mathrm{NH}_{3}{ }^{\oplus}$-Gruppen kleiner gegenüber dem der negativen $\mathrm{COO}^{\ominus}$-Gruppen, so daß beim polarisierten Molekül der Ladungsschwerpunkt mit dem Massenschwerpunkt nicht mehr zusammenfallen kann.

Inwieweit bei diesen Fragen der mögliche Einfluß elektrophoretischer Effekte noch eine zusätzliche

27 J. B. Donnet, Diss., Straßburg 1952, zitiert nach Peterlin und Stuart, 1953.

28 G. Schramm, Größe und Form von Proteinmolekülen, in: Die Phys. d. Hochpolymeren, Bd. II, S. 702-725, 1953. 
Rolle spielt, kann z. Z. nicht gesagt werden. Es ist denkbar, daß im Falle einer Teilchenwanderung bei der Entstehung der Doppelbrechung eine Komponente mitspielt, die auf einer Art „Strömungsorientierung" der Teilchen beruht. Ebenfalls ist noch unklar, welchen Einfluß der Hydratationsgrad der gelösten Teilchen auf die Doppelbrechung ausübt, da alle bisherigen Methoden zur Bestimmung des Hydratationsgrades sehr ungenaue Werte liefern und umständlich sind.

Für stetes Interesse an dieser Arbeit danke ich den Herren Professoren Dr. W. Messerschmidt und Dr. M. Klinkowski und für gewissenhafte technische Assistenz Frl. B. Lintzel.

\title{
Ubber die Wirkung von Röntgenstrahlen auf Hämoglobin
}

(Mit einer Bemerkung über die Strahlenempfindlichkeit von Gewebeschnitten)

\section{Von Otto Warburg, Walter Schröder und H. W. Gattung}

Aus dem Max-Planck-Institut für Zellphysiologie, Berlin-Dahlem

(Z. Naturforschg. 15 b, 163-166 [1960] ; eingegangen am 1. Februar 1960)

\begin{abstract}
Die Methämoglobin-Bildung durch Röntgenstrahlen erfolgt durch das bei der Bestrahlung gebildete Wasserstoffperoxyd.
\end{abstract}

Wenn Röntgenstrahlen Wasser in $\mathrm{H}+\mathrm{OH}$ spalten, so entsteht $\mathrm{H}_{2} \mathrm{O}_{2}$ durch zwei Reaktionen, durch die Reaktion

$$
\mathrm{OH}+\mathrm{OH}=\mathrm{H}_{2} \mathrm{O}_{2}
$$

und - bei Gegenwart von Sauerstoff - durch die Reaktion

$$
\mathrm{O}_{2}+2 \mathrm{H}=\mathrm{H}_{2} \mathrm{O}_{2} \text {. }
$$

Durch das so gebildete $\mathrm{H}_{2} \mathrm{O}_{2}$, nicht durch die Radikale $\mathrm{H}$ und $\mathrm{OH}$, hemmen Röntgenstrahlen die Gärung von Krebszellen, wie mit Hilfe von Katalase gezeigt worden ist ${ }^{1}$. Offenbar reagieren $\mathrm{OH}+\mathrm{OH}$ und $\mathrm{O}_{2}+2 \mathrm{H}$ so schnell, daß direkte Reaktionen von $\mathrm{H}$ oder $\mathrm{OH}$ mit den Gärungsfermenten so gut wie nicht vorkommen.

In dieser Arbeit wird für die MethämoglobinBildung durch Röntgenstrahlen gezeigt, daß auch hier die Wirkung der Strahlen über $\mathrm{H}_{2} \mathrm{O}_{2}$ geht. Am einwandfreiesten kann man dies beweisen, indem man eine neutrale Phosphatlösung ohne Hämoglobin bestrahlt und erst nach der Bestrahlung das Hämoglobin zusetzt. Dann bildet sich bei Zusatz von Hämoglobin zu der bestrahlten Lösung ebensoviel Methämoglobin, wie bei direkter Bestrahlung von Hämoglobin in der Phosphatlösung. Gibt man aber zu der bestrahlten Salzlösung zunächst Katalase - die $\mathrm{H}_{2} \mathrm{O}_{2}$ zersetzt - und erst dann das Hämoglobin, so findet man keine Bildung von Methämoglobin. Zum Beispiel betrug die prozentuale Umwandlung von Oxyhämoglobin in Methämoglobin für Hämoglobin-

1 O. Warburg u. W. Schröder, H. S. Gewitz u. W. Völker, Z. Naturforschg. 13 b, 591 [1958]. lösungen, die 0,023 $\mu \mathrm{Mol}$ Hämoglobineisen pro $\mathrm{cm}^{3}$ enthielten

\begin{tabular}{|l|c|c|}
\hline & $\begin{array}{c}\text { Röntgendosis } \\
10000 \mathrm{r} \\
\text { (in 10 Min.) }\end{array}$ & $\begin{array}{c}\text { Röntgendosis } \\
30000 \mathrm{r} \\
\text { (in 30 Min.) }\end{array}$ \\
\hline $\begin{array}{l}\text { Oxyhämoglobin in } \\
\text { Phosphat bestrahlt } \\
\text { Phosphatlösung } \\
\text { bestrahlt, dann } \\
\begin{array}{l}\text { Oxyhämoglobin } \\
\text { zugesetzt }\end{array}\end{array}$ & $\begin{array}{c}64 \% \text { Methä- } \\
\text { moglobin }\end{array}$ & $93 \%$ Methä- \\
moglobin \\
\hline
\end{tabular}

während kein Methämoglobin gefunden wurde, wenn der bestrahlten Phosphatlösung vor Zusatz des Hämoglobins Katalase zugesetzt wurde; und nur ein Bruchteil des Methämoglobins, wenn Hämoglobin unter Zusatz von Katalase bestrahlt wurde. Im ersten Fall war bei Zusatz des Hämoglobins bereits alles $\mathrm{H}_{2} \mathrm{O}_{2}$ durch die Katalase zersetzt worden. Im zweiten Fall konkurrierten Hämoglobin und Katalase während der Bestrahlung um das gebildete $\mathrm{H}_{2} \mathrm{O}_{2}$.

Wenn nun auf diese Art bewiesen ist, daß die Strahlenwirkung hier über $\mathrm{H}_{2} \mathrm{O}_{2}$ verläuft, so ergibt sich allgemein für Bestrahlungsversuche mit Hämoglobin, daß die Hämoglobin-Präparate nach Möglichkeit frei von Katalase sein sollten. Rattenhämoglobin, das bei der Hämolyse von Ratten-Erythrocyten sehr schön und schnell krystallisiert, enthält auch nach häufigem Waschen mit Wasser noch immer viel Katalase, wird aber durch mehrmaliges Umkristallisieren wesentlich katalaseärmer, wenn auch nicht frei 